\title{
Benefits of placebos
}

SIR - The gold standard for demonstrating efficacy in modern therapeutics is the placebo-controlled, double-blind (masked) clinical trial. Beatrice Golumb ${ }^{\prime}$ makes the point that it is difficult to exclude small systematic effects caused by the placebo, particularly when used over a long period. In order to try to counteract this possibility, it is the usual practice of pharmaceutical companies to employ the same formulation in both the placebo and the active treatments. Ideally, the only difference is the omission of the putative active substance. Details of both active and placebo formulations are conventionally provided to regulatory authorities in clinical trial and drug registration applications. They are rarely, if ever, published in the literature. The precaution of using similar formulations does not negate Golumb's argument. However, it is difficult to construct a test to demonstrate the inertness of a placebo. The proof involves the difficult concept of showing that the placebo never has any action, that is, proving a negative finding. Furthermore, the demonstration would itself require a placebo comparison, a situation reminiscent of the rhyme: "Dogs have fleas upon their backs to bite 'em, little fleas have lesser fleas, and so ad infinitum".

Schoemaker ${ }^{2}$, in commenting on the problems of placebo, raises several additional aspects including the perception that regulatory authorities are driving an unnecessary and inappropriate use of placebo in clinical trials ${ }^{3}$. A so-called 'pivotal' study does not necessarily involve a placebo control. It is merely a term arbitrarily used to describe the biggest, best and most convincing studies conducted to support a registration application. There are obvious areas in which the use of a placebo is unethical - infectious disease, for example. More difficult areas are diseases characterized by relapse/remission cycles, for example endogenous depression. In this situation, changes in the activity of the disease will occur, with time, in the absence of any treatment. Thus an improvement in an 'active' treatment group may be partly due to the new treatment and partly to natural remission. Without a placebo comparator, the magnitude of the two effects cannot be resolved. Any improvement in a placebotreated group, studied in parallel, allows an assessment of the relative contribution of the two effects.

Obviously, the benefit of using a placebo, in terms of improved power to predict the efficacy of drugs must be balanced against the hazards of denying patients beneficial treatment. Evidence of such denial has recently been published in studies of postoperative nausea and vomiting ${ }^{4}$, and it is probable that it also occurs in other therapeutic areas. In order to combine stringent evaluation of pharmaceutical products and correct management of patients, it would appear desirable that a transition phase should exist in the life of a drug under development, at which superiority over placebo is considered to have been demonstrated. That demonstration should act as a trigger to switch the process of evaluation to comparative studies with existing treatments. How such a switch is to be effected, whether before or after licensing, who pays for the studies, who monitors the results - all these are legitimate questions that have hardly been posed, and the answers are a long way over the horizon.

The opinions expressed above are personal, and are not necessarily shared by the board that I serve.

\section{David Lyons}

National Drugs Advisory Board,

63 Adelaide Road,

Dublin 6,

Ireland

SIR - According to Webster's Dictionary, placebo (Latin; I shall please) is a (usually pharmacologically inactive) "medicine given merely to humour the patient" without regard to disease. In this respect, placebos can become powerful tools in expert hands to relieve patients from misery and are a substantial therapeutic approach in primitive medicine. Problems may arise ${ }^{1}$ when putative therapeutic agents are tested under the misconception that placebo control is a zero-reference condition only reflecting changes related to the experimental setting.

By contrast, in otherwise controlled experiments, placebos may be as dependable a reference as the third dimension of holograms. This may particularly apply to normal and diseased brain functions, which reflect interactions of metabolic or hormonal factors and neural machinery: the action of steroids on CNS, the glucosedependent, ATP-regulated K channels linking neuron excitability and metabolic state $^{5}$, and the therapeutic effect of antidepressants through action on the hypothalamic-pituitary-adrenocortical system $^{6}$ are examples. It is true that, in these circumstances, the effect of spontaneous changes in (drug-unrelated) factors may interfere with or even mimic drug action. Biased results are a possibility in placebo-controlled trials no matter how accurate they are, and a direct control of all variables is neither realistic nor cost-effective. It is also true, however, that biased results are potentially instrumental in generating working hypotheses for further research and that progress in physiology and pharmacology should help to identify potential- ly critical, drug- or treatment-unrelated factors. Some degree of control of spontaneous variability should be possible.

The alternative to placebos (comparison with known effective drugs) may not serve its purpose either. The development of new antiepileptic drugs (once serendipitous and now based on expanding scientific opportunities but far greater effort $)^{7}$ is an example. Putative antiepileptics are first screened in clinical trials as add-on treatment for otherwise drug-resistant patients. This means test compounds are administered in association with established (standard) drugs that have already proved ineffective in any single patient and which it is hoped may prove more efficient. Ethical restraints on discontinuing treatment ${ }^{8}$ and the confounding effect of drug withdrawal motivate this approach. A modest therapeutic potency is the best expected result in these conditions, whereas adverse effects are often frequent and of ambiguous attribution.

The chance is therefore small that new antiepileptic drugs that are effective and safe for the majority of patients (who luckily are not drug-resistant) will be identified, in spite of effort, investment and labour. There are ethical counterarguments on placebo use ${ }^{8}$ yet both 'gold' standards and placebos as we know them today may be inadequate control conditions. This is not so trivial a problem in clinical pharmacology as it may seem and it is one that has long been neglected.

\section{Walter G. Sannita}

International Pharmaco-EEG Group, University Hospital San Martino,

16132 Genova,

Italy

1. Golumb, B. Nature, 375, 530 (1995).

2. Schoemaker, J. Nature 377, 98 (1995).

3. Rothman, K. \& Michels, K. New Engl. J. Med. 331 394-398 (1994).

Aspinall, R. \& Goodman, N. Br. med. J. 311, 844-846 (1995).

. Miller, R. J. Trends Neurosci. 13, 197 (1990).

6. Barden, N., Reul, J.M.H.M. \& Holsboer, F. Trends Neurosci. 18, 6 (1995).

. Financial Times, 23 March (1994)

8. Rothman, K.J. \& Michels, K.B. N. Engl. J. Med. 331 , 394 (1994).

\section{Novel satellite}

SIR - I admire Nersi Razavi's audacious plan to promote cultural tolerance by means of an orbiting double balloon, reported by Steve Nadis (Nature 377, 281; 1995). This profound experiment is clearly the first step to a new science of astrological engineering. But before funds are committed to the venture, its proposers should clarify two crucial details: (1) the mechanism by which the novel satellite will augment cultural tolerance and (2) the chance of it working.

\section{David Jones}

Department of Chemistry,

University of Newcastle,

Newcastle upon Tyne NE1 7RU, UK 